\title{
The NeST (Neoadjuvant systemic therapy in breast cancer) study: National Practice Questionnaire of United Kingdom multi- disciplinary decision making
}

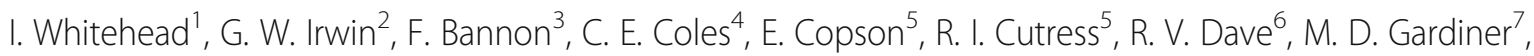
M. Grayson ${ }^{8}$, C. Holcombe ${ }^{9}$, S. Irshad ${ }^{10,11}$, C. O'Brien ${ }^{12,13}$, R. L. O'Connell ${ }^{14}$, C. Palmieri ${ }^{15,16}$, A. M. Shaaban ${ }^{17}$, N. Sharma ${ }^{18}$, J. K. Singh ${ }^{17}$, S. Potter ${ }^{19,20}$, S. A. Mclntosh ${ }^{21^{*}}$ and on behalf of the NeST Study Research Collaborative

\begin{abstract}
Background: Neoadjuvant systemic therapy (NST) is increasingly used in the treatment of breast cancer, yet it is clear that there is significant geographical variation in its use in the UK. This study aimed to examine stated practice across UK breast units, in terms of indications for use, radiological monitoring, pathological reporting of treatment response, and post-treatment surgical management.

Methods: Multidisciplinary teams (MDTs) from all UK breast units were invited to participate in the NeST study. A detailed questionnaire assessing current stated practice was distributed to all participating units in December 2017 and data collated securely usingREDCap. Descriptive statistics were calculated for each questionnaire item.

Results: Thirty-nine MDTs from a diverse range of hospitals responded. All MDTs routinely offered neoadjuvant chemotherapy (NACT) to a median of 10\% (range 5-60\%) of patients. Neoadjuvant endocrine therapy (NET) was offered to a median of $4 \%$ (range $0-25 \%$ ) of patients by $66 \%$ of MDTs. The principal indication given for use of neoadjuvant therapy was for surgical downstaging. There was no consensus on methods of radiological monitoring of response, and a wide variety of pathological reporting systems were used to assess tumour response. Twentyfive percent of centres reported resecting the original tumour footprint, irrespective of clinical/radiological response. Radiologically negative axillae at diagnosis routinely had post-NACT or post-NET sentinel lymph node biopsy (SLNB) in 73.0 and $84 \%$ of centres respectively, whereas $16 \%$ performed SLNB pre-NACT. Positive axillae at diagnosis would receive axillary node clearance at $60 \%$ of centres, regardless of response to NACT.

\footnotetext{
* Correspondence: s.mcintosh@qub.ac.uk

${ }^{21}$ Patrick G Johnston Centre for Cancer Research, Queen's University Belfast, 97 Lisburn Road, Belfast BT9 7AE, UK

Full list of author information is available at the end of the article
}

(c) The Author(s). 2021 Open Access This article is licensed under a Creative Commons Attribution 4.0 International License, which permits use, sharing, adaptation, distribution and reproduction in any medium or format, as long as you give appropriate credit to the original author(s) and the source, provide a link to the Creative Commons licence, and indicate if changes were made. The images or other third party material in this article are included in the article's Creative Commons licence, unless indicated otherwise in a credit line to the material. If material is not included in the article's Creative Commons licence and your intended use is not permitted by statutory regulation or exceeds the permitted use, you will need to obtain permission directly from the copyright holder. To view a copy of this licence, visit http://creativecommons.org/licenses/by/4.0/ The Creative Commons Public Domain Dedication waiver (http://creativecommons.org/publicdomain/zero/1.0/) applies to the data made available in this article, unless otherwise stated in a credit line to the data. 


\begin{abstract}
(Continued from previous page)
Discussion: There is wide variation in the stated use of neoadjuvant systemic therapy across the UK, with general low usage of NET. Surgical downstaging remains the most common indication of the use of NAC, although not all centres leverage the benefits of NAC for de-escalating surgery to the breast and/or axilla. There is a need for agreed multidisciplinary guidance for optimising selection and management of patients for NST. These findings will be corroborated in phase II of the NeST study which is a national collaborative prospective audit of NST utilisation and clinical outcomes.
\end{abstract}

Keywords: Breast cancer, Neoadjuvant treatment, Chemotherapy, Endocrine therapy, Surgery

\section{Background}

Breast cancer affects around 55,000 women per year in the United Kingdom, and over recent years breast cancer management has evolved, with increasing use of personalised approaches to therapy. This includes the use of neoadjuvant systemic therapy (NST), which may be used to reduce the extent of surgery, as well as to determine the sensitivity of a tumour to therapy in the in vivo setting. Long-term results from randomised control trials comparing neoadjuvant with adjuvant chemotherapy (NACT) have demonstrated no significant difference in distant recurrence, breast cancer mortality or any cause mortality [1]. Furthermore, pathological response to neoadjuvant chemotherapy has been validated as predictive of long-term outcomes, and meta-analysis has shown that approximately $\sim 26 \%$ of unselected patients can achieve a pathological complete response (pCR) [2, 3]. Pathological complete response (pCR) is higher in some disease subtypes, with for example HER2-positive breast cancers achieving pCR rates of up to $60 \%$ [4], with much lower response rates reported in ER-positive disease [2]. The presence of residual disease following neoadjuvant therapy may inform the choice of subsequent adjuvant therapies, with consequent improved outcomes [5, 6]. Furthermore, national guidance recommends offering primary systemic therapy to ER negative and HER2 positive invasive breast cancer, with international guidance recommending this approach for the treatment of stage 2 or 3 HER2 positive or triple negative disease $[7,8]$.

Primary endocrine therapy has long been used in the management of hormone receptor positive patients deemed unfit for surgery, although the role of neoadjuvant endocrine therapy (NET) is still being established. Some studies have demonstrated it to be as effective as chemotherapy in strongly hormone receptor positive disease, and the Pre-operative Endocrine Prognostic Index has been reported as a tool for use following NET to identify patients at risk of relapse [9]. The majority of trials in this setting are low powered with small sample sizes and variable treatment duration, and the extent of NET use within UK centres remains unclear [10-12].

In both Europe and the United States, significant regional variation has been reported in the use of neoadjuvant chemotherapy $[13,14]$. Similar variation has been demonstrated in the UK, in the national Mastectomy Decisions Audit (MasDA) [15]. This national prospective study showed that many centres opted for mastectomy over NST in almost 30\% of patients with large tumour to breast size ratio, missing potential opportunities for surgical downstaging. Indeed, in HER2 positive disease some $60 \%$ of patients potentially eligible for neoadjuvant therapy proceeded directly to mastectomy without such treatment in this study.

Furthermore, it has been shown that even where improved $\mathrm{pCR}$ rates can be achieved this does not necessarily impact on rates of breast conserving surgery [16]. This may be due to differences in the surgical approach to the breast following neoadjuvant therapy, with some surgeons resecting the original tumour footprint irrespective of response to treatment, whilst others will tailor their resection to residual disease. Timing of axillary staging and surgical approach to patients presenting with node-positive disease is also controversial [17]. Finally, there is little consensus in the imaging modalities used to monitor treatment response in the neoadjuvant setting, nor in pathology reporting frameworks across the UK.

This study aimed to describe the current stated practice of multidisciplinary teams (MDTs) across the UK with respect to the use of neoadjuvant systemic therapy, including indications for use, response monitoring, pathological reporting and surgical approaches to the breast and axilla. It forms the first stage of the Neoadjuvant Systemic Therapy in Breast Cancer (NeST) Study, a national multicentre, multidisciplinary collaborative prospective study assessing usage and real-world outcomes [18].

\section{Methods}

All surgical and oncological units treating breast cancer within the UK were eligible to participate in the NeST study. As with previous National Practice Questionnaires in the UK, multidisciplinary units were invited to participate by email in December 2017 via a number of professional and research organisations [19]. These included the Mammary Fold (MF) Breast Trainees' Association, the Association of Breast Surgery, the Association of 
Surgeons in Training, the National Trainee Research Collaborative (NTRC), the Breast Cancer Trainees Research Collaborative Group, the NCRI Breast Clinical Studies Group (CSG) and the Reconstructive Surgery Trials Network (RSTN). Thus each unit received an invitation to participate through several sources during December 2017, with a further reminder invitation through these routes sent in January 2018. No information was collected on units which did not respond to the invitations.

Members of the NeST steering committee developed the national practice questionnaire (NPQ), which was piloted in 4 centres and iteratively modified according to feedback, to ensure ease of use. The final 68 item questionnaire collected data on multidisciplinary team (MDT) demographics, indications for NST, proportion of patients offered NST, preferred treatment regimes, methods of monitoring response, subsequent surgical management and reporting of pathological response. The questionnaire was issued to all MDTs participating in the NeST study via the secure electronic database, REDCap in December 2017 [20], and is provided as Supplementary Data. Respondents were asked to complete the questionnaire at their weekly multidisciplinary meetings, where all specialties were present. Data was uploaded to REDCap by the local lead for the NeST study. MDTs with incomplete NPQs were contacted via e-mail and invited to input missing data in order to maximise data capture, which was completed by September 2019.

All participating institutions gained local governance approval for participation in the study.

Simple summary statistics were calculated for each questionnaire item. Categorical data were summarised by counts and percentages. Continuous data was summarised by mean, median, standard deviation and ranges as appropriate. Statistical tests were carried out using Graphpad Prism 9.

\section{Results}

Responses were obtained from a total of 39 of 144 UK MDTs. Characteristics of participating centres' service provision and MDT composition are summarised in Table 1.

\section{Indications and selection for Neoadjuvant therapy}

All MDTs in the study reported routinely offering NACT to their patients, with an estimated median of $10 \%$ (range 5-60\%) cases being offered this modality. The median usage of NACT in teaching hospitals was $10 \%$ (range $5-40 \%$ ), as compared with $7.75 \%$ in DGHs (range 5-60\%) ( $p=0.32$, Mann-Whitney U test).

Twenty-six MDTs (66\% of the total) routinely offered NET; a further $5 \%(n=2)$ offer NET only as a treatment
Table 1 Demographics of participating breast units and multidisciplinary teams

\begin{tabular}{ll}
\hline Organisation & Number (\%) \\
\hline Teaching Hospital & $23(59)$ \\
DGH & $15(38)$ \\
Not stated & $1(3)$ \\
Service Provision & \\
$\quad$ Symptomatic only & $3(8)$ \\
$\quad$ Screening/symptomatic & $36(92)$ \\
Actively recruiting to trials &
\end{tabular}

Unit size

Median cases per year (range) 470 (220-1000)

\section{MDT Composition}

Histopathologist

Radiologist

Oncologists

Clinical oncologist

Medical oncologist

Breast Surgeons

Oncoplastic Breast Surgeon

Median No. consultants (range)
$2(1-8)$
$4(1-9)$
$4(1-10)$
$2(0-5)$
$2(0-5)$
$3(0.5-10)$
$3(0.5-10)$

option within a clinical trial. For teaching hospitals, 22\% did not routinely offer NET, whereas for DGHs this figure was $27 \%$. A median of $4 \%$ (range $0-25 \%$ ) of patients were offered NET at these centres, with a median duration of treatment of 6 months (range: 3-9 months). At teaching hospitals, the median number of patients offered NET was 5\% (range $0.2-25 \%$ ), and at DGHs the corresponding figures were $2.5 \%$ (range $0.5-12.5 \%)(p=$ 0.41, Mann-Whitney U test).

Indications for recommending neoadjuvant therapy are summarised in Fig. 1. The most common indication of the use of both NACT and NET was for the downstaging of disease, either to treat locally advanced disease or to downstage planned surgery.

Neoadjuvant chemotherapy regimens most commonly reported being used are summarised in Fig. 2, with the most commonly prescribed regimen being FECdocetaxel/trastuzumab/pertuzumab for HER2-positive disease and FEC-docetaxel for HER2-negative disease. Preferred regimens were not stated in the responses from 6 MDTs (15\%).

Neoadjuvant radiotherapy use in the UK is low, with $58 \%$ of respondents reporting that they do not use this approach, and a further $38 \%$ stating that they would only use it in the context of advanced or inoperable disease unresponsive to systemic therapies and one unit only using it in the context of a clinical trial (3\%). One unit did not respond to this question. 


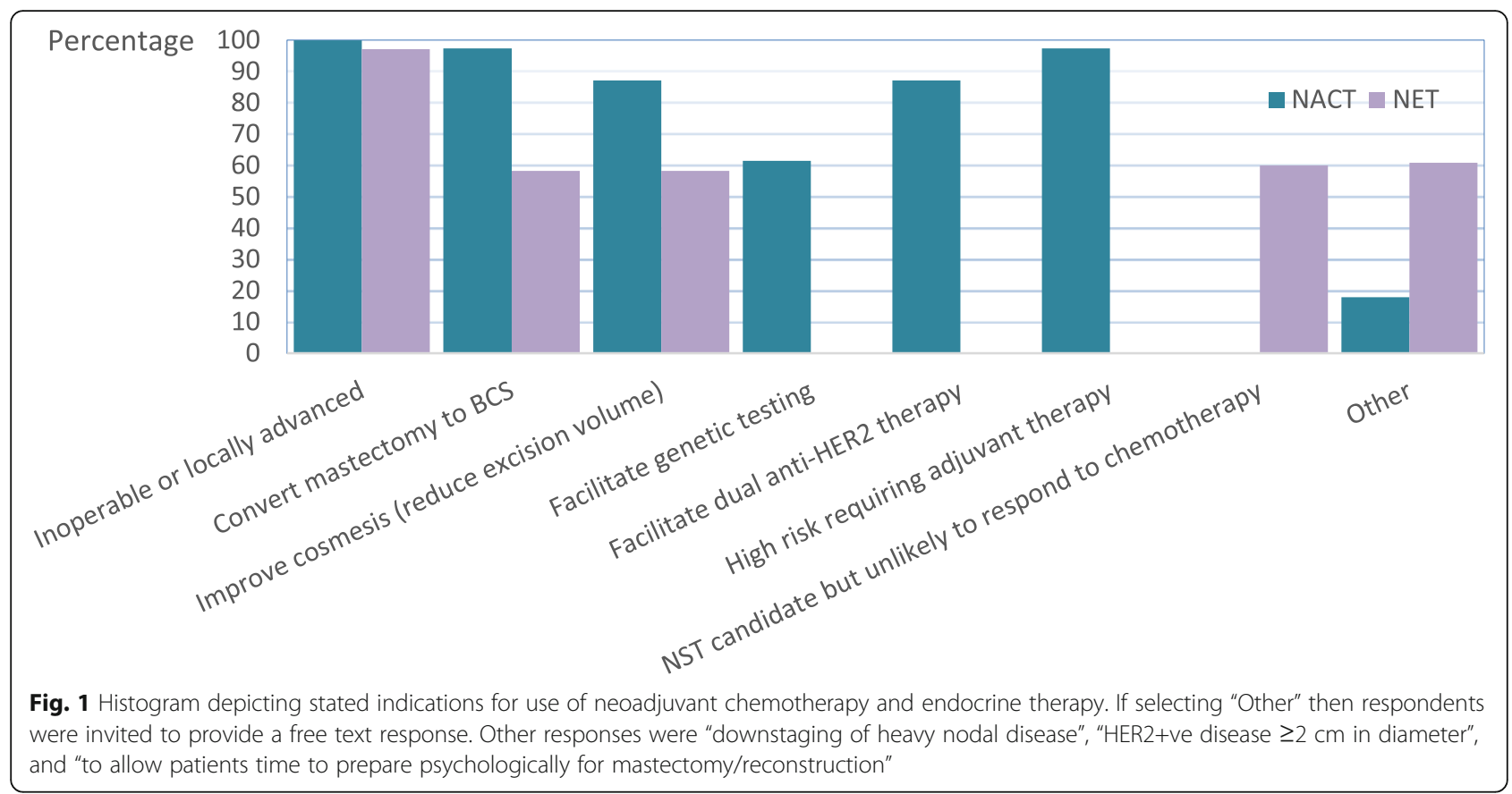

\section{a)}

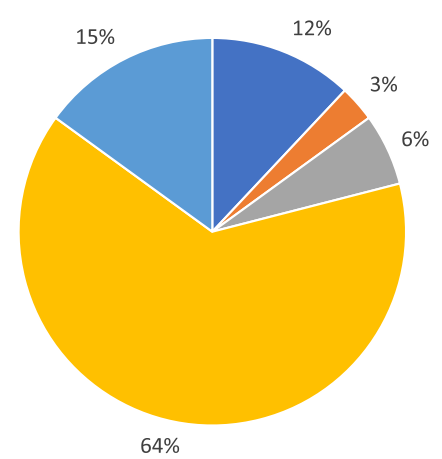

- Docetaxel/carboplatin/trastuzumab/pertuzumab

- Docetaxel/carboplatin/trastuzumab

nEC - docetaxel/trastuzumab

— FEC - docetaxel/trastuzumab/pertuzumab

a Other b)

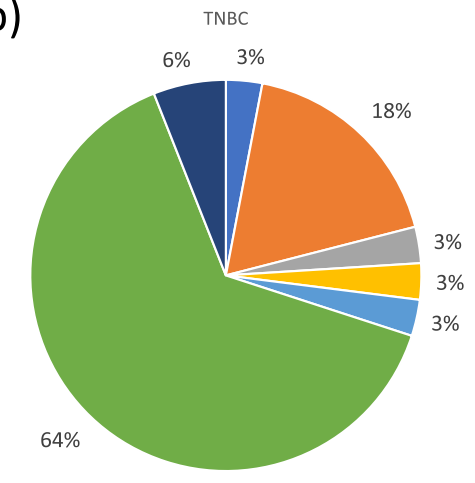

- Cyclophosphamide/docetaxel

- EC-docetaxel

- EC - dose-dense paclitaxel

- EC - docetaxel/carboplatin

- EC - paclitaxel/carboplatin

- FEC - docetaxel

- Other c)

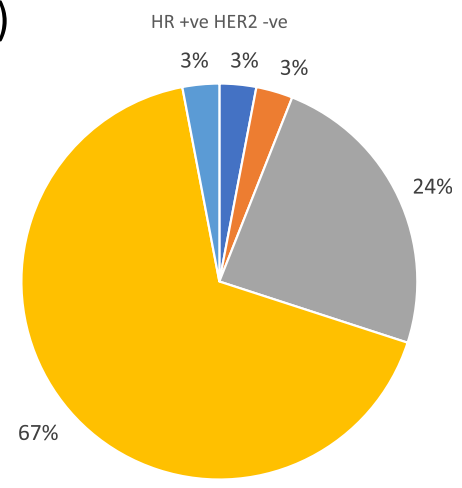

- Cyclophosphamide/docetaxel

- EC - dose-dense paclitaxel

- EC - docetaxel

- FEC - docetaxel

- Other

Fig. 2 Most commonly prescribed neoadjuvant chemotherapy regimes according to disease subtype. a HER2 positive cancer $\mathbf{b}$ Triple negative breast cancer (TNBC) c Hormone receptor positive, HER2 negative 
Monitoring and management of treatment response

Monitoring of response information was provided for all MDTs. A marker clip is routinely sited in the breast by $97 \%$ of multidisciplinary teams when using NACT. In $79 \%$ of units this is prior to commencing treatment, with $21 \%$ varying the timing due to practicalities.

Preferred modalities for monitoring response to treatment are detailed in Fig. 3 (a) and (b) for neoadjuvant chemotherapy and endocrine therapy respectively, with clinical assessment and/or ultrasound being utilised most often. Response is assessed at varying time points during treatment as follows:

- $45 \%$ mid-point and end of treatment

- $5 \%$ mid-point, end and other time point

- $3 \%$ mid-point and other time point

- $18 \%$ mid-point only

- $8 \%$ end of treatment only

- $18 \%$ other time point only

- 3\% - varies with MDT consideration of cancer and patient characteristics

A quarter of MDTs (27\%) stated they do not monitor response in patients planned to undergo mastectomy. Where response to NACT is monitored, results are routinely discussed in MDT meetings at $76 \%$ of centres with $22 \%$ of centres discussing selected patients only, and $2 \%$ (one centre) foregoing MDT discussion completely.

When using NET, $75 \%$ of MDTs site a marker clip; $86 \%$ of these centres deploy the clip prior to treatment, with $7 \%$ siting it during treatment and $7 \%$ varying the timing in response to practicalities. The median reported duration of NET was 6 months (range 3-9 months) before proceeding to surgery. All centres using NET monitor response clinically, with $95 \%$ also using radiological modalities (Fig. 3). Patients on NET are routinely discussed in $60 \%$ of MDTs, selectively discussed in $30 \%$ of MDTs and not discussed in 10\% of MDTs.

\section{Post NST loco-regional treatment}

When managing the breast post NST $74 \%$ of centres practise response-adapted surgery whereas $26 \%$ stated that they resect the original tumour footprint, regardless of the extent of clinical or radiological response to treatment. The majority of centres carry out post-NST sentinel lymph node biopsy (SLNB) in patients with clinically negative axillae at diagnosis (73\% post-NACT and $84 \%$ post-NET). In patients with clinically positive axillary nodes at diagnosis $60 \%$ of centres stated that they would carry out axillary node clearance (ANC) regardless of response to NACT, and 69\% $(n=25)$ following NET. Thirteen percent of MDTs would re-assess the axilla following NACT and 25\% following NET prior to making a surgical decision.

Post-NST, virtually all units stated that they would treate the conserved breast with adjuvant radiotherapy (97\%). Post-mastectomy radiotherapy (PMRT) was largely driven by pre-treatment tumour size and nodal status, with $92 \%$ of MDTs stating that they give PMRT where pre-treatment tumour size was $\geq 50 \mathrm{~mm}$, and $87 \%$ giving supraclavicular fossa (SCF) radiotherapy based on a pre-treatment diagnosis of $\mathrm{N} 2$ disease.

Thirty six percent of units that perform SLNB prior to NST would proceed to an ANC post-treatment, without further assessing the axilla if sentinel nodes are positive. Patients found to have a positive axilla on post-NST

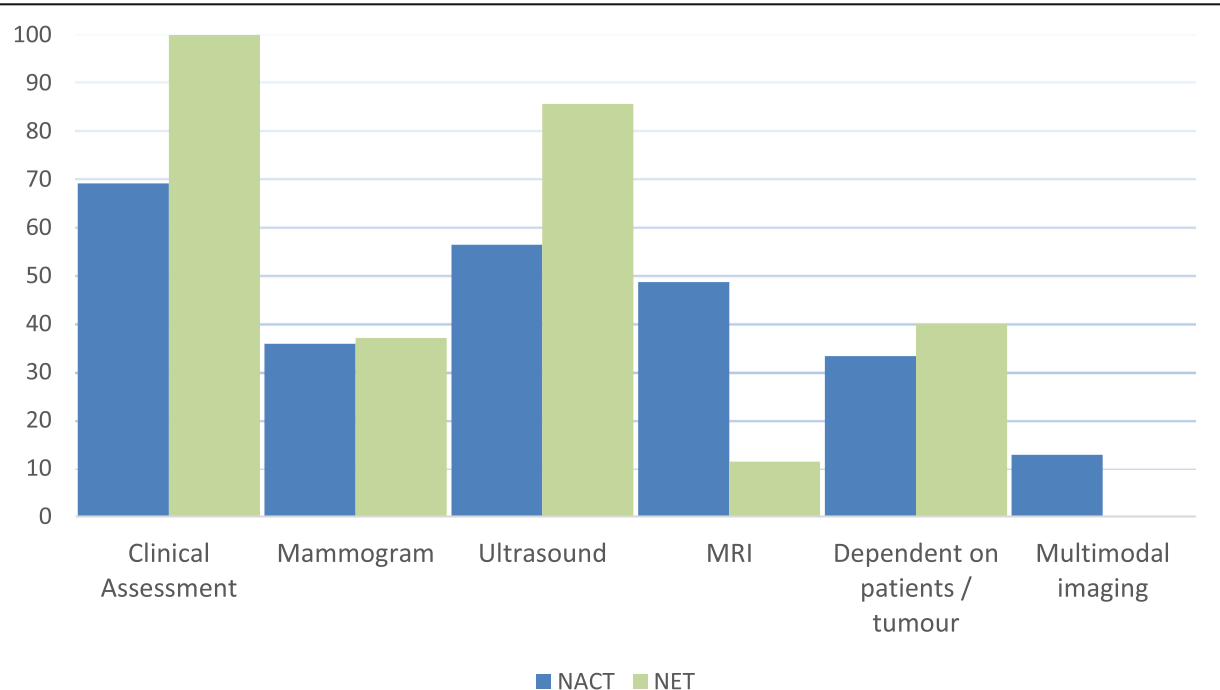

Fig. 3 Prefered modalities used in the monitoring of neoadjuvant systemic therapy response. Represented as percentage of MDTs using each modality for Fig. 3 a: neoadjuvant chemotherapy and Fig. 3 b neoadjuvant endocrine therapy. As some units use more than one imaging modality, percentages may add up to $>100 \%$ 
SLNB are managed on an individualised basis at $31 \%$ $(n=11)$ of centres following NACT and $54 \%(n=19)$ of centres following NET. Approximately half of MDTs would perform a completion ANC; $54 \%(\mathrm{n}=19)$ following NACT, and $46 \%(n=16)$ for NET.

\section{Histopathology}

In $86 \%$ of MDTs, a reporting system is routinely used to describe the extent of pathological response to NACT. Figure 4 summarises the reporting systems used, with 2 centres not responding to this question (5\%). Ki67 is routinely measured in post-NST specimens in only $11 \%$ of centres, with $8 \%$ reporting it in selected circumstances such as clinical trials, and 5\% not responding. In contrast, only $46 \%$ of MDTs use a system to report response to NET, and 14 centres use a descriptive report only.

\section{Discussion}

This study gives an overview of the stated practice in the use of neoadjuvant systemic therapy for breast cancer in the UK, confirming that wide variation in such practice exists. All 39 participating centres in the first phase of the NeST Study offer NACT, although it appears that a relatively small proportion of patients (median 10\%, range $5-60 \%)$ are recommended this treatment strategy. There is wide variation reported in the frequency with which NACT is recommended in UK MDTs, although we showed no difference in stated rates of use between teaching hospitals and DGHs, albeit in a relatively small number of hospitals using self-reported data. When considering the findings of studies such as MasDA, and considering the potential benefits for patients of using NST (both in terms of surgical downstaging and the utility of treatment response as a prognostic biomarker), it is likely that there is under-utilisation of this approach across the UK [15]. Furthermore, the variation in usage of NACT between MDTs appears too wide to be accounted for by variation in patient populations between units, although it is known that the age structure of the UK population varies by local area, and that use of both NACT and NET may consequently vary with age [21]. Individual patient-level data for patients being treated with NeST was not collected in the National Practice Questionnaire; however, the prospective audit phase of NeST will seek to explore this aspect of neoadjuvant therapy usage in more detail [18].

It remains clear that surgical downstaging is a primary indication for the use of NACT in many MDTs. However, it is equally clear that other indications for recommending NACT are emerging, in line with disease biology. Response to treatment has been shown to be a valuable predictor of long-term outcome following neoadjuvant chemotherapy [22]. However, it is also increasingly clear that pathological response to treatment can be utilised as a functional biomarker, to guide the use of subsequent adjuvant therapies where patients have an incomplete response to treatment, particularly in the context of certain disease subtypes, such as HER2+ or triple negative disease $[5,6]$.

Around two thirds of MDTs in this survey are using NET. However, this approach tends to be offered only to a small proportion of patients (a median of $4 \%$ in this study), and appears to be primarily used in the UK where disease is considered to be locally advanced or inoperable, to facilitate surgical treatment, with a relatively small proportion of centres employing this approach to downstage disease to reduce the extent of surgery. Similarly low usage of NET was seen in

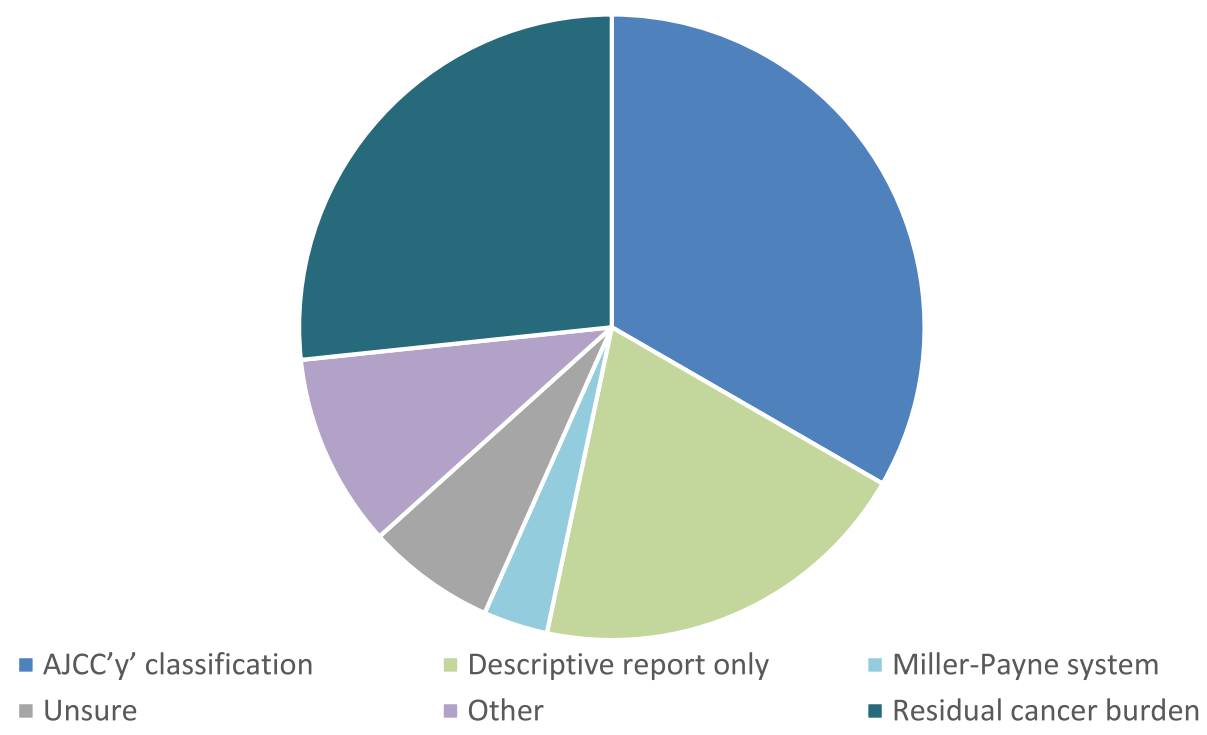

Fig. 4 Histopathology reporting systems post neoadjuvant chemotherapy 
the MasDA study, although many post-menopausal women with ER-positive breast cancer were recommended mastectomy due to a large tumour to breast size ratio, and could potentially have benefitted from NET [15]. A 2016 metanalysis suggests that NET with aromatase inhibitors is comparable to NACT in terms of radiological and clinical response rates, with similar rates of breast conserving surgery, [11]. In this meta-analysis it is noted that $90 \%$ of published studies of NET include postmenopausal women, and that there is little data on the use of NET in pre-menopausal women with hormone receptor positive breast cancer. Clearly the role and benefits of NET in such pre-menopausal women is yet to be established, and the prospective audit phase of NET will determine the age distribution and menopausal status of women in the UK being treatment with NET [18]. However, this data, taken together with the MasDA findings show that there remains a clear reluctance to utilise this approach routinely in clinical practice within the UK despite the low pCR reported with NACT in this group. The reasons for this remain unclear, but may relate to a perceived lack of evidence regarding the long-term oncological outcomes of this approach. While genomic assays may be of value here and increase clinician confidence in decision-making, there remains a need for further highquality clinical trial data to guide the management of this patient group.

It is also clear from this data that there is a wide variation in radiological monitoring and pathological reporting during and after neoadjuvant therapy, with no consensus on the optimal radiological method of monitoring response. With respect to pathological reporting, several reporting systems are available, and current UK pathology guidelines do not recommend a particular system [23]. Although the Residual Cancer Burden system is increasingly regarded as the gold standard for reporting pathological response following neoadjuvant chemotherapy and is the system recommended for neoadjuvant trials, only around one quarter of units are using this reporting system routinely, with around $20 \%$ of units issuing descriptive reports only [24].

Although surgical downstaging was noted to be a key indication for recommending NAC in this study, around 25\% of centres stated that following treatment, the surgical goal remained removal of the original tumour footprint, regardless of response. This is in contrast to the St Gallen consensus guidance, which recommended that excision of the initial tumour bed was not required [25]. It seems likely, therefore, that the opportunity to de-escalate breast surgery following NACT is not being fully utilised in some patients.

Management of the axilla following NACT has been a controversial area, although subsequent to this survey, UK multidisciplinary recommendations have been produced to guide treatment [26]. At the time of this study, the majority of patients diagnosed with clinically node negative breast cancer receiving NACT were undergoing post-treatment SLNB. However, in patients presenting with node-positive disease, the majority of centres were performing axillary node clearance, with only a small number of centres carrying out axillary reassessment and response-guided treatment of the axilla. It appears likely, therefore, that a proportion of patients may not have the opportunity for de-escalation of axillary surgery following NACT, although the impact of the published guidance remains to be seen.

Clearly, there are some limitations to the data provided by this questionnaire. Only 39 of 144 breast units (27\%) in the UK participated in the study, and consequently there may be selection bias as these units may not be representative of UK practice more broadly. Although this data has been supplied by all members of the MDT and therefore should represent an accurate reflection of multidisciplinary perspectives, we accept that this study is based on reported rather than actual practice of MDTs. We note that the self-reported range of NACT use is from 5 to $60 \%$, with the upper limit appearing surpriginly high. While this may reflect genuine variation in NACT use (and that some centres, including specialist tertiary referral centres are high users of this treatment approach), it is possible that selfreported data may not be entirely reflective of actual patterns of care. Consequently the data may be reflective of perceived rather than actual practice, as studies (albeit in other specialties) have demonstrated there to be a difference between these [27]. Furthermore, units were not specifically asked about compliance with national and international guidelines regarding the use of NeST such as UK NICE guidance or the St Gallen guidelines [7, 8]. However, the prospective audit phase of the NeST study will provide valuable insight into decision-making on an individual patient basis across UK MDTs [18].

In spite of these limitations, to our knowledge this is the first UK study to broadly examine real-world stated practice in terms of the use of neoadjuvant systemic therapy. It is clear that there is wide variety in perceived indications for and use of neoadjuvant systemic therapy in the UK, as well as a lack of consensus on the optimal methods for monitoring and reporting response and on the surgical management of the primary tumour following systemic therapy. Although NICE guidance outlines potential indications of the use of NST in breast cancer, this study indicates a clear need for both further research and the development of multidisciplinary guidance with respect to monitoring of response, pathological reporting and surgical decision-making, to ensure optimal outcomes for breast cancer patients treated with neoadjuvant therapies. We await the prospective phase of the NeST study, which will allow the corroboration of these results with the real-world use of neoadjuvant systemic therapy for breast cancer in the UK [18]. 


\section{Appendix}

Table 2 NeST Study Collaborators

\begin{tabular}{|c|c|c|}
\hline Liz & Clayton & Royal Surrey County Hospital \\
\hline Ellen & Copson & University Hospitals Southampton NHS Trust \\
\hline Karina & Cox & Maidstone and Tunbridge Wells \\
\hline Tim & Crook & Royal Surrey County Hospital \\
\hline Beatrix & Elsberger & Ninewells Hospital Dundee \\
\hline Ahmed & Ghoneima & Queen Alexandra Hospital Portsmouth \\
\hline Sirwan & Hadad & Royal Hallamshire Hospital Sheffield \\
\hline Anita & Hargreaves & Warrington \& Halton NHS Trust \\
\hline Paul & Healy & St Vincent's Hospital Dublin \\
\hline Adam & Heetun & University Hospitals Southampton NHS Trust \\
\hline Dan & Henderson & Heart of England NHS Trust \\
\hline Julia & Henderson & Royal Liverpool University Hospital \\
\hline Natalie & Hirst & Bradford Teaching Hospitals \\
\hline Fiona & Hoar & City Hospital Birmingham \\
\hline Mike & Hughes & Airedale General Hospital \\
\hline Emma & Iddles & Aberdeen Royal Infirmary \\
\hline Sheeba & Irshad & Guys Kings \& St Thomas' \\
\hline Tracey & Irvine & Royal Surrey County Hospital \\
\hline Stacey & Jones & St James' University Hospital Leeds \\
\hline Sarantos & Kaptanis & Bedford Hospital \\
\hline Emma & Maclnnes & Doncaster Royal Infirmary \\
\hline Andrew & McCanny & Ulster Hospital Dundonald \\
\hline Linda & McLaughlin & \\
\hline Anthony & Neal & Royal Surrey County Hospital \\
\hline Rachel & O'Connell & Royal Surrey County Hospital \\
\hline Neill & Patani & Royal Marsden Hospital \\
\hline Belinda & Pearce & Royal Hampshire County Hospital \\
\hline Mandana & Pennick & Betsi Cadwaladr - Glan Clywd hospital \\
\hline Simon & Pilgrim & University Hospitals Leicester \\
\hline Rene & Roux & Oxford University Hospitals NHS Trust \\
\hline Matthew & Rowland & Royal Devon \& Exeter \\
\hline Sunita & Saha & Colchester General Hospital \\
\hline Kavita & Sharma & Glasgow Royal Infirmary \\
\hline Jagdeep & Singh & University Hospitals Birmingham \\
\hline Chiara & Sirianni & Betsi Cadwaladr \\
\hline Brendan & Skelly & Belfast City Hospital \\
\hline Rachel & Soulsby & Milton Keynes University Hospital \\
\hline Mark & Tatterton & Salisbury District Hospital \\
\hline Rob & Thomas & Royal Victoria Infirmary Newcastle \\
\hline Medy & Tsalic & Heart of England NHS Trust \\
\hline Raghavan & Vidya & Royal Wolverhampton NHS Trust \\
\hline Olivia & Waker & Royal Surrey County Hospital \\
\hline Lisa & Whisker & Nottingham University Hospitals NHS Trust \\
\hline $\operatorname{lan}$ & Whitehead & St. Helens and Knowsely Teaching Hospitals \\
\hline Janet & Woods & Heart of England NHS Trust \\
\hline
\end{tabular}

\section{Abbreviations}

ABS: Association of Breast Surgery of Great Britain and Ireland; ANC: Axillary node clearance.; DGH: District General Hospital.; MasDA: Mastectomy Decisions Audit;; MDT: Multidisciplinary team.; MF: Mammary Fold.;

NACT: Neoadjuvant chemotherapy;i NCRI: National Cancer Research Institute.; NeST: Neoadjuvant systemic therapy.; NET: Neoadjuvant endocrine therapy.; NICE: National Institute for Health and Care Excellence.; pCR: Pathological complete response.; RSTN: Reconstructive Surgery Trials Network: SLNB: Sentinel lymph node biopsy.

\section{Acknowledgments}

Not applicable.

\section{Authors' contributions}

IW wrote the first draft of the manuscript. Gl, CEC, EC, TIC, RVD, MG, CH SI, COB, ROC, CP, AS, NS, JS and IW all contributed to study design, including design of the data collection instruments, and to the writing and editing of the protocol. FB contributed to study design, methodology and the statistical analysis plan. GI, ROC, SP and IW undertook piloting of the data collection instruments. SMcl and SP were responsible for the conception of the project, and contributed to the design of the project, and to the writing and editing of the protocol. All listed collaborators were involved in the collection and upload of data from individual MDTs for analysis. All authors have read and approved the final version of the manuscript.

\section{Funding}

The NeST Study was funded by a grant from the Association of Breast Surgery. The funder has had no role in the design of the study nor collection of data, and will have no input into the analysis or interpretation of the data.

\section{Availability of data and materials}

The datasets generated during and/or analysed during the current study (the NeST National Practice Questionnaire) may be made available upon request from Stuart McIntosh (s.mcintosh@qub.ac.uk). Requests for access to the data will be reviewed by the NeST Study Steering Group prior to any data sharing.

\section{Ethics approval and consent to participate}

This study reported in this manuscript was a survey of medical professionals to determine stated practice for the use of neoadjuvant systemic therapy in breast cancer. Bothe this survey and the ongoing prospective cohort study will not affect clinical care. Consequently, research ethics approval is not required and this has been confirmed by the Health Research Authority online decision tool (http://www.hra-decisiontools.org.uk/research/). A Trainee Lead was identified at each participating unit, who in turn identified a named supervising consultant to act as the principal investigator for registration purposes. The study lead, in consultation with the principal investigator to registered the audit at each site and obtained local audit approvals for study participation prior to commencing data collection. A copy of local approval is held by the NeST study team. Patient consent is not required, as no patient identifiable data are being recorded and there is no risk to patients.

\section{Consent for publication}

Not applicable, as outlined above; no patient identifiable data is collected or published.

\section{Competing interests}

None declared.

\section{Author details}

${ }^{1}$ Royal Liverpool University Hospital, Liverpool University Hospitals NHS Foundation Trust, Prescot Street, Liverpool L7 8XP, UK. '²elfast Health and Social Care Trust, Belfast City Hospital, Lisburn Road, Belfast BT9 7AB, UK. ${ }^{3}$ Centre for Public Health, Queen's University Belfast, Institute of Clinical Science, Block A, Royal Victoria Hospital, Belfast BT12 6BA, UK. ${ }^{4}$ University of Cambridge, Cambridge, UK. ${ }^{5}$ Cancer Sciences Academic Unit, Faculty of Medicine, University of Southampton, Southampton SO16 6YD, UK. ${ }^{6}$ The Nightingale Centre, Wythenshawe Hospital, Manchester University NHS Foundation Trust, Manchester M23 9LT, UK. ${ }^{7}$ Department of Plastic Surgery, 
Wexham Park Hospital, Frimley Health NHS Foundation Trust, Slough SL2 $4 \mathrm{HL}$, UK. ${ }^{8} \mathrm{NI}$ Cancer Research Consumer Forum, c/o NI Cancer Trials Network, East Podium, C-Floor, Belfast City Hospital, Belfast BT9 7AB, UK. ' ${ }^{9}$ Liverpool University Hospitals Foundation Trust, Prescot Street, Liverpool L7 8XP, UK. ${ }^{10}$ Guy's Cancer Centre, Guy's \& St Thomas' NHS Trust, Great Maze Pond, London SE1 9RT, UK. ${ }^{11}$ School of Cancer \& Pharmaceutical Sciences, King's College London, London SE1 9RT, UK. ${ }^{12}$ The Christie Hospital NHS Foundation Trust, Wilmslow Road, Manchester M20 2BX, UK. ${ }^{13}$ School of Medical Sciences Faculty of Biology, Medicine and Health University of Manchester, Manchester M13 9PL, UK. ${ }^{14}$ Royal Marsden NHS Foundation Trust, Downs Road, Sutton, Surrey SM2 5PT, UK. ${ }^{15}$ University of Liverpool, Institute of Systems, Molecular and Integrative Biology, Department of Molecular and Clinical Cancer Medicine, Liverpool, UK. ${ }^{16}$ The Clatterbridge Cancer Centre NHS Foundation Trust, Liverpool, UK. ${ }^{17}$ Queen Elizabeth Hospital Birmingham and University of Birmingham, Mindelsohn Way, Edgbaston, Birmingham B15 2GW, UK. ${ }^{18}$ Breast Unit, Level 1 Chancellor wing, St James Hospital, Beckett Street, Leeds LS97TF, UK. ${ }^{19}$ Bristol Centre for Surgical Research, Population Health Sciences, Bristol Medical School, Canynge Hall, 39 Whatley Road, Clifton, Bristol BS8 2PS, UK. ${ }^{20}$ Bristol Breast Care Centre, North Bristol NHS Trust, Southmead Hospital, Southmead Road, Bristol BS10 5NB, UK. ${ }^{21}$ Patrick G Johnston Centre for Cancer Research, Queen's University Belfast, 97 Lisburn Road, Belfast BT9 7AE, UK.

Received: 4 August 2020 Accepted: 21 December 2020 Published online: 22 January 2021

\section{References}

1. Alberro JA, Ballester B, Deulofeu P, Fabregas R, Fraile M, Gubern JM, et al. Long-term outcomes for neoadjuvant versus adjuvant chemotherapy in early breast cancer: meta-analysis of individual patient data from ten randomised trials. Lancet Oncol. 2018;19(1):27-39.

2. Cortazar P, Zhang L, Untch M, Mehta K, Costantino JP, Wolmark N, et al. Pathological complete response and long-term clinical benefit in breast cancer: the CTNeoBC pooled analysis. Lancet. 2014;384(9938):164-72.

3. Spring LM, Fell G, Arfe A, Sharma C, Greenup R, Reynolds KL, et al. Pathologic complete response after Neoadjuvant chemotherapy and impact on breast Cancer recurrence and survival: a comprehensive meta-analysis. Clin Cancer Res. 2020;26(12):2838-48.

4. Schneeweiss A, Chia S, Hickish T, Harvey V, Eniu A, Hegg R, et al. Pertuzumab plus trastuzumab in combination with standard neoadjuvant anthracycline-containing and anthracycline-free chemotherapy regimens in patients with HER2-positive early breast cancer: a randomized phase II cardiac safety study (TRYPHAENA). Annals Oncol. 2013;24(9):2278-84.

5. von Minckwitz G, Huang CS, Mano MS, Loibl S, Mamounas EP, Untch M, et al. Trastuzumab Emtansine for Residual Invasive HER2-Positive Breast Cancer. N Engl J Med. 2019;380:617-28.

6. Masuda N, Lee S-J, Ohtani S, Im Y-H, Lee E-S, Yokota I, et al. Adjuvant Capecitabine for breast Cancer after preoperative chemotherapy. N Engl J Med. 2017;376(22):2147-59.

7. National Institute for Health \& Care Excellence. Early and Locally Advanced Breast Cancer: Diagnosis and Management 2018 [Available from: https:// www.nice.org.uk/guidance/ng101/chapter/Recommendations\#primarysystemic-therapy.

8. Burstein HJ, Curigliano G, Loibl S, Dubsky P, Gnant M, Poortmans P, et al. Estimating the benefits of therapy for early-stage breast cancer: the St. Gallen international consensus guidelines for the primary therapy of early breast cancer 2019. Annals Oncol. 2019;30(10):1541-57.

9. Ellis MJ, Tao Y, Luo J, A'Hern R, Evans DB, Bhatnagar AS, et al. Outcome prediction for estrogen receptor-positive breast cancer based on postneoadjuvant endocrine therapy tumor characteristics. J Natl Cancer Inst. 2008;100(19):1380-8

10. Palmieri C, Cleator S, Kilburn LS, Kim SB, Ahn SH, Beresford M, et al. NEOCENT: a randomised feasibility and translational study comparing neoadjuvant endocrine therapy with chemotherapy in ER-rich postmenopausal primary breast cancer. Breast Cancer Res Treat. 2014;148(3): 581-90.

11. Spring LM, Gupta A, Reynolds KL, Gadd MA, Ellisen LW, Isakoff SJ, et al. Neoadjuvant endocrine therapy for estrogen receptor-positive breast Cancer: a systematic review and meta-analysis. JAMA Oncol. 2016;2(11): 1477-86.
12. Arthur LM, Turnbull AK, Khan LR, Dixon JM. Pre-operative endocrine therapy Curr Breast Cancer Rep. 2017;9(4):202-9.

13. Spronk PER, van Bommel ACM, Siesling S, Wouters M, Vrancken Peeters M, Smorenburg $\mathrm{CH}$. Variation in use of neoadjuvant chemotherapy in patients with stage III breast cancer: results of the Dutch national breast cancer audit. Breast. 2017;36:34-8.

14. Mougalian SS, Soulos PR, Killelea BK, Lannin DR, Abu-Khalaf MM, DiGiovanna MP, et al. Use of neoadjuvant chemotherapy for patients with stage I to III breast cancer in the United States. Cancer. 2015;121(15):2544-52.

15. Mastectomy Decisions Audit Collaborative obotWMRC. Multicentre prospective observational study evaluating recommendations for mastectomy by multidisciplinary teams. Br J Surg. 2020;107(3):227-37.

16. Criscitiello C, Curigliano G, Burstein HJ, Wong S, Esposito A, Viale G, et al. Breast conservation following neoadjuvant therapy for breast cancer in the modern era: are we losing the opportunity? Eur J Surg Oncol. 2016;42(12): 1780-6.

17. Pilewskie M, Morrow M. Axillary nodal management following Neoadjuvant chemotherapy: a review. JAMA Oncol. 2017;3(4):549-55.

18. Irwin GW, Bannon F, Coles CE, Copson E, Cutress RI, Dave RV, et al. The NeST (neoadjuvant systemic therapy in breast cancer) study - protocol for a prospective multi-Centre cohort study to assess the current utilization and short-term outcomes of neoadjuvant systemic therapies in breast cancer. Int J Surg Protoc. 2019;18:5-11.

19. Mylvaganam S, Conroy E, Williamson PR, Barnes NLP, Cutress RI, Gardiner $M D$, et al. Variation in the provision and practice of implant-based breast reconstruction in the UK: Results from the iBRA national practice questionnaire. Breast. 2017;35(Supplement C):182-190.

20. Harris PA, Taylor R, Thielke R, Payne J, Gonzalez N, Conde JG. Research electronic data capture (REDCap)--a metadata-driven methodology and workflow process for providing translational research informatics support. J Biomed Inform. 2009;42(2):377-81.

21. Office of National Statistics. Overview of the UK population: 20192019 [Available from: https://www.ons.gov.uk/peoplepopulationandcommunity/ populationandmigration/populationestimates/articles/ overviewoftheukpopulation/august2019.

22. Symmans WF, Wei C, Gould R, Yu X, Zhang Y, Liu M, et al. Long-term prognostic risk after Neoadjuvant chemotherapy associated with residual Cancer burden and breast Cancer subtype. J Clin Oncol. 2017;35(10):1049-60.

23. Pinder SE, Rakha EA, Purdie CA, Bartlett JM, Francis A, Stein RC, et al. Macroscopic handling and reporting of breast cancer specimens pre- and post-neoadjuvant chemotherapy treatment: review of pathological issues and suggested approaches. Histopathology. 2015;67(3):279-93.

24. Symmans WF, Peintinger F, Hatzis C, Rajan R, Kuerer $H$, Valero V, et al. Measurement of residual breast cancer burden to predict survival after neoadjuvant chemotherapy. J Clin Oncol. 2007;25(28):4414-22.

25. Curigliano G, Burstein HJ, Winer EP, Gnant M, Dubsky P, Loibl S, et al. Deescalating and escalating treatments for early-stage breast cancer: the St. Gallen international expert consensus conference on the primary therapy of early breast Cancer 2017. Annals Oncol. 2017;28(8):1700-12.

26. Gandhi A, Coles C, Makris A, Provenzano E, Goyal A, Maxwell AJ, et al. Axillary surgery following Neoadjuvant chemotherapy - multidisciplinary guidance from the Association of Breast Surgery, Faculty of Clinical Oncology of the Royal College of Radiologists, UK breast Cancer group, National Coordinating Committee for breast pathology and British Society of Breast Radiology. Clin Oncol (R Coll Radiol). 2019;31(9):664-8.

27. Gill KV, Voils SA, Chenault GA, Brophy GM. Perceived versus actual sedation practices in adult intensive care unit patients receiving mechanical ventilation. Ann Pharmacother. 2012;46(10):1331-9.

\section{Publisher's Note}

Springer Nature remains neutral with regard to jurisdictional claims in published maps and institutional affiliations. 